\title{
All-fiber all-normal-dispersion femtosecond laser with a nonlinear multimodal interference-based saturable absorber
}

\author{
UĞUr TeĞın ${ }^{1,2, *}$ (1) AND BüLEND Ortaç ${ }^{1,2,3}$ \\ ${ }^{1}$ National Nanotechnology Research Center, Bilkent University, 06800 Bilkent, Ankara, Turkey \\ ${ }^{2}$ Institute of Materials Science and Nanotechnology, Bilkent University, 06800 Bilkent, Ankara, Turkey \\ ${ }^{3}$ e-mail: ortac@unam.bilkent.edu.tr \\ *Corresponding author: ugurtegin@unam.bilkent.edu.tr
}

Received 12 January 2018; revised 1 March 2018; accepted 2 March 2018; posted 5 March 2018 (Doc. ID 319516$) ;$ published 30 March 2018

In this Letter, we demonstrate, to the best of our knowledge, the first all-fiber all-normal-dispersion ytterbium-doped oscillator with a nonlinear multimodal interference-based saturable absorber capable of generating ultrashort dissipative soliton pulses. Additional to functioning as a saturable absorber, the use of multimode fiber segments between single-mode fibers also ensures the bandpass filtering via multimode interference reimaging necessary to obtain dissipative soliton mode locking. The oscillator generates dissipative soliton pulses at $1030 \mathrm{~nm}$ with $5.8 \mathrm{~mW}$ average power, 5 ps duration, and $44.25 \mathrm{MHz}$ repetition rate. Pulses are dechirped to $276 \mathrm{fs}$ via an external grating compressor. All-fiber cavity design ensures high stability, and $\sim 70 \mathrm{~dB}$ sideband suppression is measured in the radio frequency spectrum. Numerical simulations are performed to investigate cavity dynamics, and obtained results are well matched with experimental observations. The proposed cavity presents an alternative approach to achieve all-fiber dissipative soliton mode locking with a simple and low-cost design. () 2018 Optical Society of America

OCIS codes: (060.3510) Lasers, fiber; (140.4050) Mode-locked lasers; (320.7090) Ultrafast lasers; (190.4370) Nonlinear optics, fibers.

https://doi.org/10.1364/OL.43.001611

Fiber-based laser systems are important for material processing, medical applications, and optical metrology [1]. Ytterbiumbased fiber lasers are generally preferred in the photonic systems due to high and broadband gain of the ytterbium active ions. Various mode-locking dynamics are proposed in the literature to obtain ultrashort pulses from ytterbium-based fiber cavities with soliton [2], dispersion managed [3], and self-similar pulses $[4,5]$. However, a certain degree of dispersion mapping inside the laser cavity is necessary to achieve stable mode-locking operation in these types of fiber lasers. At $1 \mu \mathrm{m}$ wavelength range, negative dispersion can be implemented by bulk grating or photonic crystal fibers, which results in increased complexity and undermines benefits of fiber lasers such as compactness and stability. Later, a stable passively mode-locked all-normaldispersion fiber laser is demonstrated, and pulse generation is attributed to the strong spectral filtering of chirped pulses [6]. Over the last decade, all-normal-dispersion fiber lasers have been studied extensively by exploiting dissipative soliton pulse dynamics [7]. In the literature, the power and energy scalability of the dissipative soliton pulses is demonstrated with very-largemode-area fibers $[8,9]$. On the other hand, all-fiber dissipative soliton lasers are a subject of high interest owing to their compact and misalignment-free designs. In the literature, researchers demonstrate different all-fiber oscillators by proposing several methods to obtain in-line all-fiber bandpass filtering necessary to achieve dissipative soliton pulses at $1 \mu \mathrm{m}$ wavelength range [10-13]. Nonlinear polarization evolution (NPE) and material-based saturable absorbers are generally preferred to start mode-locked operation in these studies.

In the last few years, graded-index multimode fibers have become subject to the extensive study of nonlinear optics due to their unique properties. In the literature, researchers reported new nonlinear dynamics such as spatiotemporal instability [14-16], supercontinuum generation [17-19], self-beam cleaning [17,20,21], multimode solitons [22], and their dispersive waves [23]. In addition to the aforementioned studies, saturable absorber behavior of a short graded-index multimode fiber segment is theoretically proposed by Nazemosadat $e t$ al. [24]. In the proposed method, in between single-mode fiber segments, a graded-index multimode fiber can be used to obtain nonlinear multimodal interference (NL-MMI) to achieve a saturable absorber attribute. The results of Nazemosadat $e t$ al. indicate a saturable absorber can be obtained with more than $90 \%$ modulation depths via the proposed structure. On the other hand, an all-fiber bandpass filter is later demonstrated with similar device structure both numerically and experimentally [25]. The proposed filter is based on the multimode interference reimaging phenomenon. Later, Mafi et al. presented a low-loss wavelength-dependent coupler structure by implementing a graded-index multimode fiber segment to this filter structure [26]. Very recently, the use of a short gradedindex multimode fiber segment as a saturable absorber was 


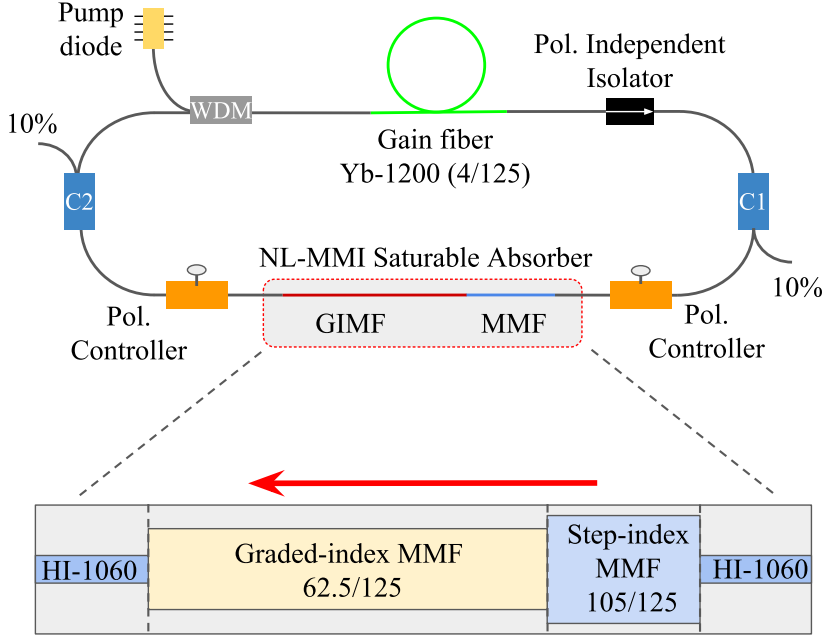

Fig. 1. Schematic of the all-fiber Yb-doped laser with NL-MMIbased saturable absorber: WDM, wavelength division multiplexer; GIMF, graded-index multimode fiber; MMF, multimode fiber.

experimentally verified in a thulium based all-fiber soliton oscillator with a slightly modified structure [27]. In between the single mode and graded-index multimode fibers, Li et al. placed a step-index multimode fiber to ensure excitation of the highorder modes in the graded-index multimode fiber and achieved saturable absorber behavior. They reported a soliton all-fiber oscillator capable of generating $1.4 \mathrm{ps}$ pulses at $1888 \mathrm{~nm}$ with $19.82 \mathrm{MHz}$ repetition rate and $0.6 \mathrm{~mW}$ output average power. Additionally, they systematically studied the effect of step-index and graded-index multimode fiber lengths in the proposed saturable absorber structure to the mode-locking threshold and output power of the laser. The proposed NL-MMI-based saturable absorber configuration also supports bandpass filter structure; thus, it is very promising for all-normal-dispersion lasers to obtain dissipative soliton pulses with simple and all-fiber-cavity design. However, to the best of our knowledge, there are no reports on employing the NL-MMI-based saturable absorber and bandpass filter configuration of a multimode fiber segment in an all-normal-dispersion mode-locked fiber laser to realize a dissipative-soliton operation.

Here, we present the first all-fiber integrated all-normaldispersion $\mathrm{Yb}$-doped oscillator with the NL-MMI-based saturable absorber capable of generating ultrashort pulses both numerically and experimentally. With this simple cavity design, a compact, stable, and low-cost dissipative soliton fiber laser is presented. Numerical simulations investigate the possibility of dissipative soliton mode locking and lead to experimental studies by revealing the internal cavity dynamics. The experimentally demonstrated oscillator is self-starting and generates dissipative solitons at $1030 \mathrm{~nm}$ with $5.8 \mathrm{~mW}$ average power and $44.25 \mathrm{MHz}$ repetition rate. Chirped dissipative soliton pulses are compressed externally to $276 \mathrm{fs}$.

The oscillator scheme is presented in Fig. 1. The length of the multimode fibers used in the saturable absorber segment is important for mode-locking threshold and bandwidth of the bandpass filter. As illustrated in Fig. 1, we prefer to use the step-index multimode fiber $(105 / 125)$ to graded-index multimode fiber $(62.5 / 125)$ configuration as described by Li et al.
[27] for adequate excitation of the graded-index multimode fiber. To obtain $\sim 15 \mathrm{~nm}$ bandwidth for multimode interferencebased bandpass filtering, the length of the graded-index multimode fiber is determined as $14 \mathrm{~cm}$ [25,26]. With a $0.5 \mathrm{~cm}$ step-index multimode fiber segment, the total length of the NL-MMI-based saturable absorber segment is defined as $14.5 \mathrm{~cm}$. The transmission behavior of the NL-MMI-based saturable absorber is presented as sinusoidal by Nazemosadat $e t$ al. [24]. According to Nazemosadat et al., the modulation depth and minimum transmission of the saturable absorber segment are defined by the length of the graded-index multimode fiber and energy splitting between the modes; thus, for a constant fiber length, one can achieve desired modulation depth.

We first performed numerical simulations based on the model described in [28-30] to investigate the possibility of dissipative soliton mode locking with the proposed cavity (Fig. 1). Based on Nazemosadat et al.'s study, we modeled the saturable absorber segment by a transfer function of the form $T=$ $q_{0}+q_{1} \sin ^{2}\left(I / I_{\text {sat }}\right)$, where $q_{0}=0.15$ is the minimum transmission, $q_{1}=0.85$ is the modulation depth, $I$ is instantaneous
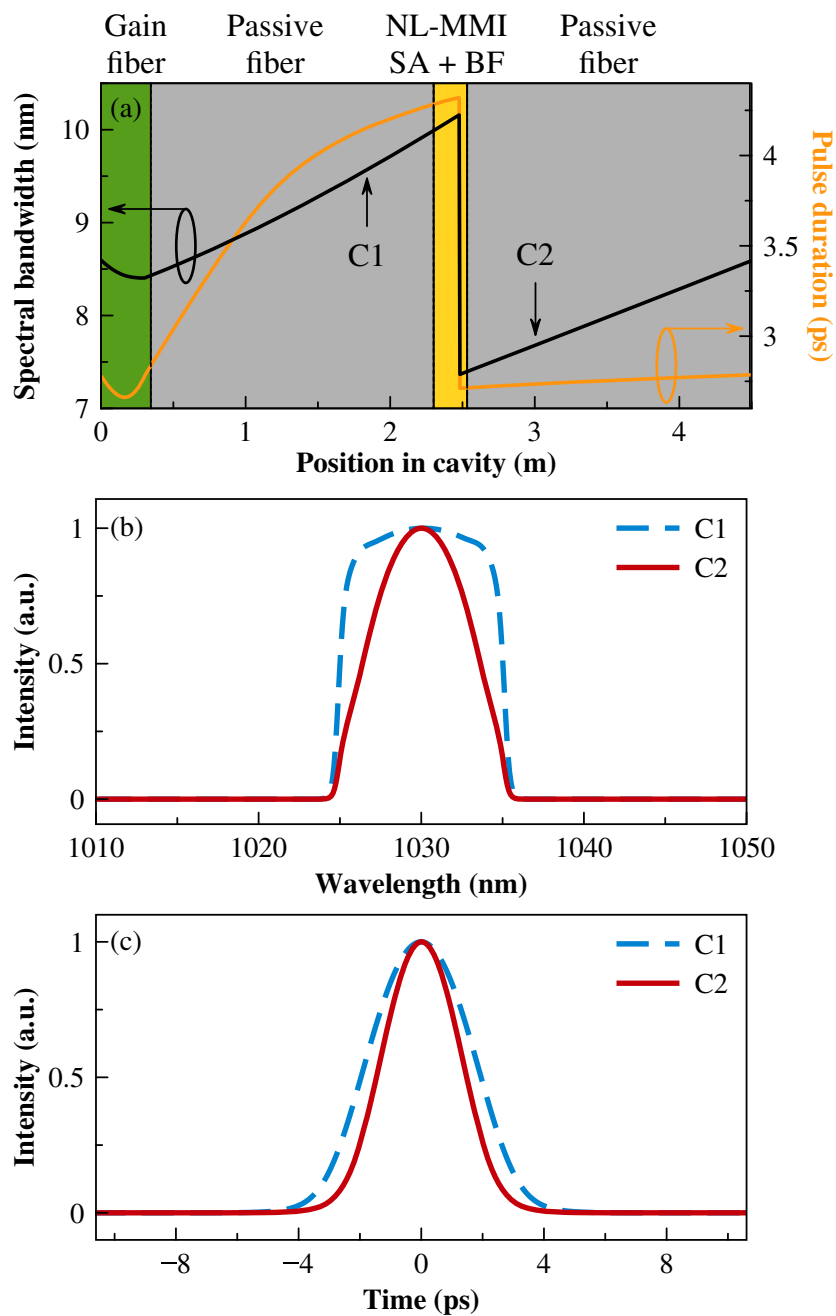

Fig. 2. (a) Simulated pulse duration and spectral bandwidth variation over the cavity: SA, saturable absorber; BF, bandpass filter. (b) Simulated laser spectra obtained from the defined output couplers. C1 (C2) is defined before (after) the gain fiber segment. (c) Simulated temporal profile obtained at the output couplers $\mathrm{C} 1$ and $\mathrm{C} 2$. 

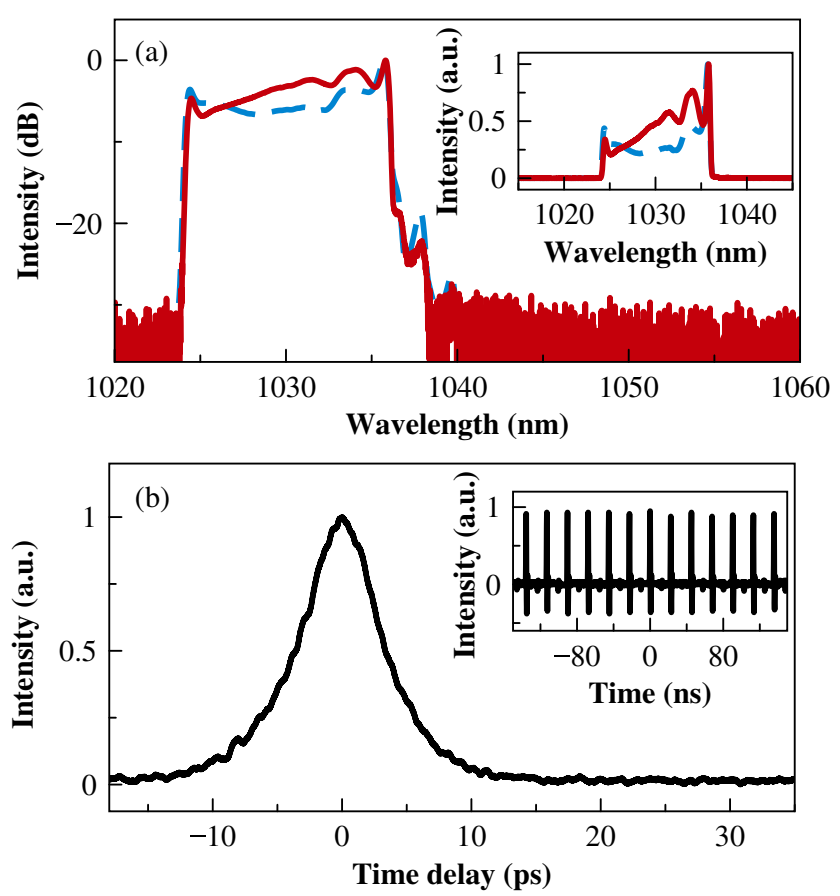

Fig. 3. Spectra of dissipative soliton pulse from the output couplers on logarithmic (a) and linear scale (inset). Measurements from C1 and $\mathrm{C} 2$ couplers are indicated as blue and red, respectively. (b) Autocorrelation trace of the chirped pulses obtained directly from output coupler C1. Inset: single-pulse train.

pulse power, and $I_{\text {sat }}=0.4 \mathrm{~nJ}$ is the saturation power [24]. In the NL-MMI saturable absorber, the graded-index multimode fiber light propagates into a single-mode fiber; therefore, the energy in the higher-order modes acts as an additional intracavity loss. Thus, in the simulations, we consider pulse propagation only in the fundamental mode and introduce an additional loss term at the saturable absorber segment. This loss term mimics the energy content of the high-order modes that cannot propagate in the following single-mode fiber segment. By comparing the fiber core sizes of the graded-index multimode and singlemode fibers, we defined this loss term as 0.95 in our simulations. With the $14 \mathrm{~cm}$ graded-index multimode fiber segment of the cavity, the multimode interference bandpass filter is defined as a Gaussian filter with $15 \mathrm{~nm}$ bandwidth according to calculations presented by Mafi et al. [26]. In simulations, the saturable absorber transfer function, bandpass filtering, and the loss term are introduced at the end of the graded-index multimode fiber. The gain is defined as Lorentzian with $40 \mathrm{~nm}$ bandwidth and $30 \mathrm{~dB}$ small-signal gain. Raman scattering and shock terms are also incorporated in our numerical studies.

The numerical simulations are performed with fourth-order Runge-Kutta in the interaction picture method [30,31]. The initial field is defined as a quantum noise. A stable mode-lock regime is found, and the results are demonstrated in Fig. 2. At the first output port after the gain fiber (C1), dissipative soliton pulses with $1.3 \mathrm{~nJ}$ intracavity pulse energy are obtained. Intracavity evolution of the pulse width in the spectral and temporal domains is presented in Fig. 2(a). We notice that gain filtering shortens the spectral width in the first part of the active fiber, whereas the spectrum is broadened by Kerr nonlinearity in the following passive fiber segment. The required pulse
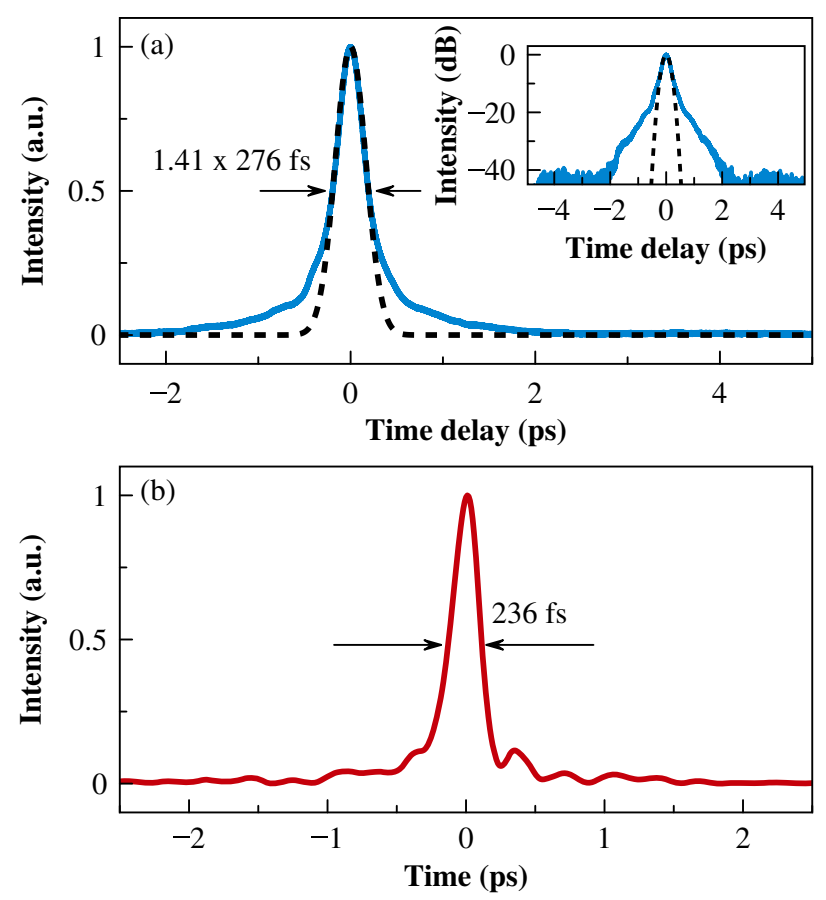

Fig. 4. (a) Autocorrelation trace of the dechirped pulses obtained from output coupler C1 (solid) and theoretical fit with Gaussian pulse shape (dashed). Inset: in logarithmic scale. (b) PICASO retrieved dechirped pulse shape.

shaping based on chirping and spectral filtering is acquired by a multimode interference-based bandpass filter segment. The relatively high intracavity loss due to multimode to single-mode fiber propagation and strong amplification supports the self-consistent dissipative soliton evolution. For output couplers before and after the gain fiber, the spectra have $10 \mathrm{~nm}$ and $7.3 \mathrm{~nm}$ bandwidths, respectively [Fig. 2(b)]. As presented in Fig. 2(c), the output pulse durations of 3.9 ps and 2.9 ps are obtained, respectively, for these couplers. The same numerical solution is reached from different initial quantum noise fields.

The experiments are then performed with the cavity design determined by the numerical simulations. The gain section of the oscillator is a $30 \mathrm{~cm}$ highly doped ytterbium fiber ( $\mathrm{Yb}$ 1200-4/125) pumped by a $976 \mathrm{~nm}$ fiber-coupled diode laser. An inline isolator is employed to determine pulse propagation direction inside the ring cavity. To eliminate the possibility of NPE mode locking, we intentionally prefer to use a polarization independent isolator. As defined by the simulations, $10 \%$ output couplers are placed before and after the NL-MMI-based saturable absorber segment to investigate intracavity pulse dynamics. The NL-MMI saturable absorber segment is developed by a $0.5 \mathrm{~cm}$ step-index multimode fiber with $105 \mu \mathrm{m}$ core and $125 \mu \mathrm{m}$ cladding diameter (Nufern MM-S105/125) and a $14 \mathrm{~cm}$ graded-index multimode fiber with $62.5 \mu \mathrm{m}$ core and $125 \mu \mathrm{m}$ cladding diameter (Thorlabs GIF625). Fiber polarization controllers are used for ensuring optimum intra-cavity birefringence to achieve mode-locking operation. The self-starting fundamental mode locking of a single-pulse train with a repetition rate of $\sim 44 \mathrm{MHz}$ is observed around $400 \mathrm{~mW}$ pump power. Measured optical spectra from the output couplers are presented in Fig. 3(a), and the central 


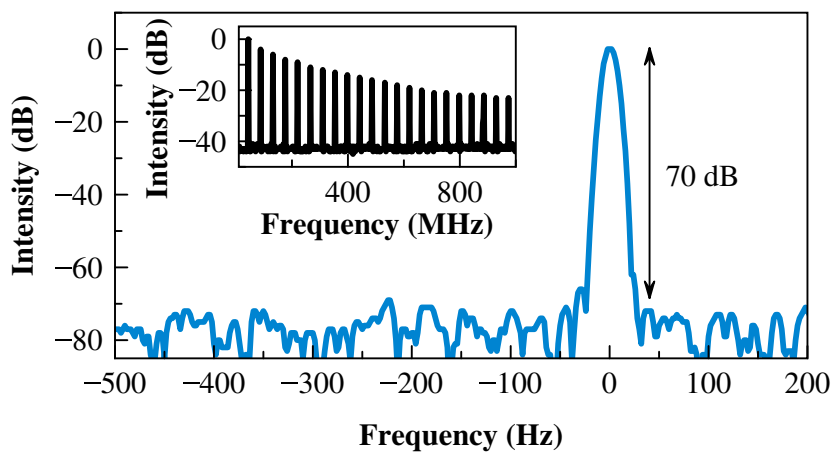

Fig. 5. Measured RF spectrum with $1 \mathrm{kHz}$ span and $10 \mathrm{~Hz}$ resolution bandwidth, with central frequency shifted to zero for clarity. Inset: RF spectrum with $1 \mathrm{GHz}$ span and $3 \mathrm{kHz}$ resolution bandwidth.

wavelengths of the pulses are recorded at $1030 \mathrm{~nm}$. Spectral bandwidths are recorded as $11.8 \mathrm{~nm}$ and $11.4 \mathrm{~nm}$ at $-7 \mathrm{~dB}$ from the couplers $\mathrm{C} 1$ and $\mathrm{C} 2$, respectively. Based on this observation, the pulse breathing ratio in one round trip is close to unity, which is typical behavior of dissipative solitons in all-normal-dispersion fiber lasers. The output power of the laser is measured as $5.8 \mathrm{~mW}$ and $0.15 \mathrm{~mW}$ at the couplers $\mathrm{C} 1$ and $\mathrm{C} 2$, respectively. As we expect from the numerical simulations, the intracavity pulse energy of $1.3 \mathrm{~nJ}$ is obtained experimentally before the output coupler $(\mathrm{C} 1)$. The temporal characterizations are performed for the pulses obtained from the $\mathrm{C} 1$ output coupler. The laser produces chirped pulses with $\sim 5$ ps duration as presented in the autocorrelation trace in Fig. 3(b). These chirped pulses are compressed externally using a grating compressor with a 300 line $/ \mathrm{mm}$ diffraction grating pair. The minimum pulse duration of $276 \mathrm{fs}$ is obtained with a Gaussian deconvolution factor and demonstrated in Fig. 4(a). To retrieve the temporal profile of the compressed pulses from the autocorrelation and spectrum data, we employ the PICASO algorithm [32]. The PICASO-retrieved shape of the pulse is presented in the Fig. 4(b). The algorithm indicates the retrieved pulse duration is $236 \mathrm{fs}$.

We characterized the oscillator in the frequency domain as well. The fundamental repetition rate of the laser is verified with a with radio frequency (RF) analyzer as $44.25 \mathrm{MHz}$. With $1 \mathrm{kHz}$ span and $10 \mathrm{~Hz}$ resolution bandwidth, we measured the RF spectrum and observed a sideband suppression ratio around $70 \mathrm{~dB}$ (Fig. 5). The fiber laser has outstanding stability both in the short and long term. The laser continues the mode-locking operation uninterrupted for days, and there is no sign of degradation. The measured RF spectrum with $1 \mathrm{GHz}$ span and $3 \mathrm{kHz}$ resolution bandwidth indicates no periodic envelope or fluctuation observed in the long span.

In conclusion, we numerically and experimentally demonstrate an all-fiber all-normal-dispersion ytterbium-doped oscillator with a nonlinear multimodal interference-based saturable absorber capable of generating ultrashort dissipative soliton pulses, for the first time in the literature. Via multimode interference reimaging, the preferred saturable absorber structure also ensures the bandpass filtering necessary to obtain dissipative soliton pulses. Thus, the proposed cavity presents an alternative approach to achieve all-fiber dissipative soliton mode locking with the simple and low-cost design. The oscillator generates dissipative soliton pulses at $1030 \mathrm{~nm}$ with $5.8 \mathrm{~mW}$ average power, $0.13 \mathrm{~nJ}$ energy, 5 ps duration, and $44.25 \mathrm{MHz}$ repetition rate. Pulses are dechirped to $276 \mathrm{fs}$ via an external grating compressor. All-fiber cavity design ensures high stability. The lownoise operation of the all-fiber cavity is confirmed, and $\sim 70 \mathrm{~dB}$ sideband suppression is measured in the RF spectrum. We believe the reported all-fiber oscillator configuration is an attractive solution for generating ultrashort dissipative soliton pulses and can find application in supercontinuum generation and optical metrology.

\section{REFERENCES}

1. M. E. Fermann and I. Hartl, Nat. Photonics 7, 868 (2013).

2. A. Isomäki and O. G. Okhotnikov, Opt. Express 14, 9238 (2006).

3. B. Ortaç, A. Hideur, T. Chartier, M. Brunel, C. Özkul, and F. Sanchez, Opt. Lett. 28, 1305 (2003).

4. J. Dudley, A. Peacock, V. Kruglov, B. Thomsen, J. Harvey, M. Fermann, G. Sucha, and D. Harter, Optical Fiber Communication Conference (Optical Society of America, 2001), paper WP4.

5. F. Ilday, J. Buckley, W. Clark, and F. Wise, Phys. Rev. Lett. 92, 213902 (2004).

6. A. Chong, J. Buckley, W. Renninger, and F. Wise, Opt. Express 14, 10095 (2006).

7. P. Grelu and N. Akhmediev, Nat. Photonics 6, 84 (2012).

8. B. Ortaç, M. Baumgartl, J. Limpert, and A. Tünnermann, Opt. Lett. 34, 1585 (2009).

9. M. Baumgartl, C. Lecaplain, A. Hideur, J. Limpert, and A. Tünnermann, Opt. Lett. 37, 1640 (2012).

10. K. Kieu and F. Wise, Opt. Express 16, 11453 (2008).

11. M. Schultz, H. Karow, O. Prochnow, D. Wandt, U. Morgner, and D. Kracht, Opt. Express 16, 19562 (2008).

12. K. Özgören and F. Ilday, Opt. Lett. 35, 1296 (2010).

13. L. Zhang, J. Hu, J. Wang, and Y. Feng, Opt. Lett. 37, 3828 (2012).

14. K. Krupa, A. Tonello, A. Barthélémy, V. Couderc, B. M. Shalaby, A. Bendahmane, G. Millot, and S. Wabnitz, Phys. Rev. Lett. 116, 183901 (2016).

15. L. G. Wright, Z. Liu, D. A. Nolan, M.-J. Li, D. N. Christodoulides, and F. W. Wise, Nat. Photonics 10, 771 (2016).

16. U. Teğin and B. Ortaç, IEEE Photon. Technol. Lett. 29, 2195 (2017).

17. G. Lopez-Galmiche, Z. S. Eznaveh, M. Eftekhar, J. A. Lopez, L. Wright, F. Wise, D. Christodoulides, and R. A. Correa, Opt. Lett. 41, 2553 (2016).

18. K. Krupa, C. Louot, V. Couderc, M. Fabert, R. Guenard, B. Shalaby, A. Tonello, D. Pagnoux, P. Leproux, A. Bendahmane, R. Dupiol, G. Millot, and S. Wabnitz, Opt. Lett. 41, 5785 (2016).

19. U. Teğin and B. Ortaç, "High power high repetition rate supercontinuum generation in graded-index multimode fibers," arXiv:1709.09158 (2017).

20. K. Krupa, A. Tonello, B. M. Shalaby, M. Fabert, A. Barthélémy, G. Millot, S. Wabnitz, and V. Couderc, Nat. Photonics 11, 237 (2017).

21. Z. Liu, L. G. Wright, D. N. Christodoulides, and F. W. Wise, Opt. Lett. 41, 3675 (2016)

22. W. H. Renninger and F. W. Wise, Nat. Commun. 4, 1719 (2013).

23. L. G. Wright, D. N. Christodoulides, and F. W. Wise, Nat. Photonics 9 , 306 (2015).

24. E. Nazemosadat and A. Mafi, J. Opt. Soc. Am. B 30, 1357 (2013).

25. W. S. Mohammed, P. W. Smith, and X. Gu, Opt. Lett. 31, 2547 (2006).

26. A. Mafi, P. Hofmann, C. J. Salvin, and A. Schülzgen, Opt. Lett. 36, 3596 (2011)

27. H. Li, Z. Wang, C. Li, J. Zhang, and S. Xu, Opt. Express 25, 26546 (2017).

28. G. P. Agrawal, Nonlinear Fiber Optics (Academic, 2007).

29. G. P. Agrawal, Applications of Nonlinear Fiber Optics (Academic, 2001).

30. C.. Șenel, "33 femtosecond Yb-doped optical frequency comb for frequency metrology applications," Ph.D. dissertation (Bilkent University, 2013).

31. J. Hult, J. Lightwave Technol. 25, 3770 (2007).

32. J. Nicholson, J. Jasapara, W. Rudolph, F. Omenetto, and A. Taylor, Opt. Lett. 24, 1774 (1999). 